\title{
How many quanta are there in a quantum spacetime?
}

\section{Seramika Ari Wahyoedi*}

Centre de Physique Theorique de Marseille

E-mail: ceramyca@gmail.com

\begin{abstract}
We develop a technique for describing quantum states of the gravitational field in terms of coarse grained spin networks, following earlier insights by Livine and Terno [1], [2]. This technique shows that the number of nodes and links in a quantum state of gravity, and the spin associated to the links, depend on the observables chosen for the description of the state, and therefore the question of the title is ill posed, unless further information about what is been precisely measured is given.

References

[1] S.Ariwahjoedi, J. S. Kosasih, C. Rovelli, F. P. Zen, How many quanta are there in a quantum spacetime? [gr-qc/1404.1750]

[2] E. R. Livine and D. R. Terno, Bulk entropy in loop quantum gravity, Nuclear Physics B 794 (May, 2008) 138-153, [gr-qc/0706.0985].

[3] E. R. Livine and D. R. Terno,Reconstructing Quantum Geometry from Quantum Information: Area Renormalisation, Coarse-Graining and Entanglement on Spin Networks, [gr-qc/0603008].

[4] D. Colosi and C. Rovelli,What is a particle?, Class. Quant. Grav. 26 (2009) 25002, [grqc/0409054].
\end{abstract}

Frontiers of Fundamental Physics 14 - FFP14,

15-18 July 2014

Aix Marseille University (AMU) Saint-Charles Campus, Marseille

\footnotetext{
${ }^{*}$ Speaker.
} 